le portiQue Le Portique

Revue de philosophie et de sciences humaines

$6 \mid 2000$

Le discours universitaire

\title{
Un discours éclaté
}

Entre logique disciplinaire et logique curriculaire

\section{Maurice Sachot}

\section{OpenEdition}

Journals

Édition électronique

URL : http://journals.openedition.org/leportique/432

DOI : $10.4000 /$ leportique.432

ISSN : $1777-5280$

Éditeur

Association "Les Amis du Portique"

Édition imprimée

Date de publication : 1 septembre 2000

ISSN : 1283-8594

Référence électronique

Maurice Sachot, «Un discours éclaté », Le Portique [En ligne], 6 | 2000, mis en ligne le 24 mars 2005,

consulté le 25 mars 2021. URL : http://journals.openedition.org/leportique/432 ; DOI : https://doi.org/

10.4000/leportique.432

Ce document a été généré automatiquement le 25 mars 2021.

Tous droits réservés 


\title{
Un discours éclaté
}

\author{
Entre logique disciplinaire et logique curriculaire
}

\section{Maurice Sachot}

1 Parce que la maîtrise du discours et de la pensée a partie liée avec le pouvoir et l'intérêt, parce que, plus fondamentalement, l'exercice de la pensée implique sa constante remise en cause, le discours universitaire est, par définition, non seulement un discours critique mais encore un discours en crise. Dire, par conséquent, qu'il est actuellement en crise est un truisme. Mais telle n'est pas la nature du bouleversement que connaît présentement l'Université. La dynamique qui l'entraîne à une vitesse qui va s'accélérant remet radicalement en cause le paradigme même sur lequel elle se fonde. Le discours disciplinaire qu'elle tient en interne subit les assauts du discours curriculaire que, de l'extérieur, on tient sur elle et qu'on lui demande de cautionner de par l'autorité que, justement, lui donne la science, nécessairement disciplinaire. Il y a là une contradiction qui est en même temps une sérieuse menace.

I. Le paradigme disciplinaire

2 Un universitaire se définit par sa discipline. Le mot a de moins en moins bonne presse. Le champ disciplinaire, l'interdisciplinarité, la multidisciplinarité, la transdisciplinarité, voire la transversalité seraient, pense-t-on, des catégories autrement plus pertinentes et appropriées pour aborder et traiter les questions que l'on se pose aujourd'hui ou, plutôt, les questions telles qu'on se les pose aujourd'hui, c'est-à-dire en les considérant plus volontiers dans leur complexité. C'est d'ailleurs ce qui se passe dans bien des «facultés professionnelles», comme, par exemple, celles de médecine et de droit, et cela, apparemment, à la satisfaction générale. Pourquoi n'en serait-il pas ainsi dans toutes les sciences, y compris les sciences humaines?

3 Nous ne prétendrons pas que la question que nous posons est résolue pour la médecine et le droit et qu'elle ne se présente plus que pour les autres sciences, avant tout les sciences humaines. Nous serions même enclin à nous demander si la question ne mérite pas d'être inversée : ne serait-ce pas plutôt à la médecine et au droit de rejoindre l'épistémologie qui fonde les autres sciences? Ne serait-il pas opportun de revenir sur la répartition qui s'est faite dès la formation des Universités, entre la médecine, le droit et les lettres, la première se donnant le corps physique pour objet, le second le corps 
social, le troisième ensemble, enfin, la pensée ? C'est dans ce dernier groupe, qui ne se ramène pas aux lettres au sens actuel du mot, mais qui englobe, outre la théologie, la philosophie et les lettres proprement dites, toutes les artes qui donneront naissance, bien au-delà des disciplines académiques, aux multiples disciplines et sciences actuelles, y compris les sciences exactes, que la question d'une épistémologie proprement dite s'est posée avec le plus d'acuité. Elles se sont en effet constituées non seulement en se dégageant de toute visée utilitariste directe, mais encore en se donnant positivement la connaissance comme unique objet. Il leur a fallu, pour cela, acquérir leur indépendance par rapport à tous les pouvoirs (politiques, économiques et financiers) et, plus fondamentalement encore, se libérer d'une épistémologie de la révélation qui régnait en Occident depuis que le christianisme s'était façonné comme religion d'empire. Combat inachevé et toujours à recommencer, aujourd'hui peut-être plus qu'hier. Les sciences ont donc toujours à lutter pour acquérir et maintenir la seule logique qui les fonde et que porte le terme de discipline : la logique de scientificité.

1. Discipline de recherche

4 Discipline. La consonance latine du mot pourrait faire croire que la logique scientifique qu'il évoque et les pratiques qu'il recouvre nous viennent en droite ligne de la philosophie grecque : discipline reprend disciplina, qui lui-même reprend épistèmè. Ce serait une erreur. Certes, c'est bien fondamentalement la même logique de scientificité qui fut élaborée et par les savants grecs de l'Antiquité et par les savants modernes. Et il y a bien un lien entre les deux. Mais il y a eu solution de continuité entre les deux périodes. La science moderne fut une redécouverte et, plus encore, une reconquête, jamais définitivement acquise, sur la prétention de l'institution à statuer sur le vrai.

5 Peut-être est-ce d'ailleurs cette rupture qu'enregistre d'abord le français " disciplines » (au pluriel). Dans le langage chrétien, disciplina est synonyme de religio et de doctrina. Il définit l'institué ecclésial non seulement en tant que règle de vie (regula) personnelle et collective, mais également en tant que doctrine révélée (doctrina) et démarche personnelle d'adhésion irrévocable (fides). Cette configuration résulte, comme nous avons essayé de le montrer dans L'Invention du Christ: genèse d'une religion (Odile Jacob, 1998), des métamorphoses qu'a connues le mouvement inauguré par Jésus en fonction des espaces culturels et linguistiques dans lesquels il s'est implanté et, donc, repensé. La définition nouvelle que ce mouvement donne à religio, lorsqu'il s'énonce en latin à l'extrême fin du second siècle, retient surtout de la première période, celle de l'élaboration en milieu sémitique, la dynamique de l'adhésion personnelle (fides) en réponse au "message évangélique », de la seconde période, celle de la réélaboration en milieu hellénistique, il conserve surtout la formulation de cette foi en termes philosophiques (Christ devient Logos, mais la démarche philosophique et scientifique est retournée pour être mise au service de l'adhésion croyante, pistis). La reprise de religio à son profit, enfin, alors qu'il est classé par les Latins dans la catégorie de superstitio (survivance religieuse illégitime), lui permet d'investir sur ce terme l'acquis des deux étapes précédentes et de s'incorporer l'institué romain que désigne précisément religio. La religion chrétienne se construit alors comme une socio-structure holistique dans laquelle l'institué commande à la pensée et, par la pensée, à l'individu, tout en affirmant qu'il repose sur l'individu et la pensée, s'interdisant ainsi l'enlisement fondamentaliste et donnant prise à une possible séparation et autonomisation de chacune de ces trois composantes. Ce qui finira par advenir. Ce mouvement, toujours en cours, s'effectue selon deux logiques principales : celle de la sécularisation et celle de la laïcisation. $\mathrm{Si}$, donc, le discours chrétien peut distinguer, en son sein, diverses 
disciplinae, diverses artes, notamment toutes celles qui sont liées au langage, à la parole et à l'herméneutique, celles-ci ne peuvent avoir que le statut d'ancillae, de servantes totalement soumises et n'ayant aucune sorte d'autonomie. Aussi bien, une investigation plus poussée sur l'histoire du mot discipline dans la langue française montrerait vraisemblablement que son apparition dans le champ de la pensée accompagne, voire spécifie la rupture ou, plus exactement, le retournement du paradigme chrétien de l'énonciation de la vérité, la distinction nette entre les trois instances qu'il a intégrées : la primauté est désormais donnée à l'individu (stade évangélique), lequel jouit de la liberté de penser (stade hellénistique) et de décider de l'institution (stade romain). Disciplinae apparaît en effet comme synonyme de scientiae, au sens moderne de sciences, c'est-à-dire comme élaboration d'une énonciation personnelle dont la prétention à dire du vrai ne dépend que d'une démarche humaine et, plus précisément, d'une certaine mise en œuvre de la raison.

C'est en cela et à ce moment-là seulement que le terme disciplines redécouvre et retrouve en quelque sorte l'épistèmè grecque, considérée comme fondatrice de la pensée scientifique en Occident. Avec peut-être quelques nuances qui méritent d'être signalées. Dans la pensée des philosophes et des savants grecs, le modèle épistémologique à l'œuvre est, nous semble-t-il, celui d'une recherche fondamentale et "pure ", celle qui ne vise qu'à la connaissance du réel, sans que cette connaissance implique une modification de la réalité, ni par la recherche elle-même ni par ses conséquences ou applications. Le véritable savant fait de la recherche pour la recherche, comme on dit que l'on fait de l'art pour l'art. Ce qui implique que cette recherche est absolument libre, comme la faculté d'en faire connaitre les résultats, que la connaissance et le savoir qu'elle produit n'ont d'autre autorité que celle que leur confère la démarche de scientificité, sans dépendre d'une autorité institutionnelle ni lui en conférer, que la liberté de penser est une prérogative de tout un chacun, c'est-à-dire de tout homme, quel qu'il soit, savant ou non, et non le privilège de quelques-uns.

7 Cette liberté par rapport à toute forme d'orthodoxie ne dégage pas pour autant le chercheur de la responsabilité personnelle et collective qui est la sienne. Elle l'engage, au contraire, et, cela, sous deux formes principales: d'abord vis-à-vis de l'objet de la recherche, du réel lui-même. Selon l'épistémologie grecque, le chercheur s'abstient de modifier le monde (dont les personnes font évidemment partie) pour le connaître. Il le respecte. De manière absolue. Il se contente de le questionner, travaillant sur ce que l'on dit du monde et sur les représentations qu'on en a. "Examinons de près ce que nous disons ", aime à répéter Socrate (par exemple, Eutyphron, 7a). Ensuite vis-à-vis de lui-même et des autres quant aux résultats : il les soumet à son jugement avant de les communiquer ou d'en tirer parti. Primum non nocere, dit le fameux précepte hippocratique que nous reprenons sous sa forme latine. D'abord ne pas nuire. L'épistémologie grecque est donc en même temps, sous ce double aspect, une éthique. Et sur le plan personnel, la trace de cette activité du logos est moins perçue sous le mode de la transformation que d'une mise en harmonie, en soi et par rapport au monde, en fonction d'une taxinomie que l'on a soi-même élaborée. Ce n'est pas en tant que philosophe, mais de politikos anèr, d'homme politique, que Platon a écrit des œuvres comme la République et s'est engagé dans l'action. Il nous semble que c'est ce même modèle épistémologique qui est au travail dans la scientificité qui s'élabore avec la science moderne, modèle d'autant plus marqué que, à la différence de son moment 
grec, il s'oppose au modèle prégnant d'une épistémologie dogmatique et institutionnelle, qu'il soit chrétien ou sécularisé.

8 Cette prise de position cache cependant une nuance de taille: la présence d'une épistémologie latine que le christianisme n'a pas abolie. À la différence du Grec, que la langue pousse à saisir les choses par la médiation conceptuelle et explicite de leur essence, le Latin est concret. Si le Grec cherche l'archè, l'origine ou, peut-être plus exactement, l'originaire qui, à la fois, transcende le monde sensitif et l'histoire, le latin se situe d'emblée dans le monde des choses (res) et de leur cours. Il ne les quitte jamais. S'il y a une transcendance, c'est dans l'immanence du monde qu'elle réside, dans la capacité reconnue au monde et aux hommes de se construire eux-mêmes. C'est à l'homme que revient de déterminer, de dire (fatum, que la traduction par destin assimile à tort à tychè), de manière arbitraire, dans une linéarité indéterminée, le moment précis où il fait commencer sa propre histoire, son initium (lequel n'est pas davantage à assimiler à l'archè des Grecs). S'il y eut jamais un âge d'or, puis un âge d'argent, l'âge romain - l'histoire réelle - commence avec l'âge d'airain (aeneus), c'està-dire avec l'âge du métal qui fait violence aux autres (arma) et aux choses (le socle de l'araire qui creuse le sillon qui délimite l'espace - cf. le récit fondateur, fratricide, de Rome - ou féconde le champ (la sédentarisation). L'homme idéal (le vir), dans cette perspective, est alors un héros, construisant sa propre destinée comme celle de sa cité. L'histoire est un récit de gesta, de facta, bien situés dans le temps (elle prend la forme d'Annales), non un débat d'idées. Le poète a parfaitement compris sa propre culture, lorsqu'il nomme son héros fondateur de Rome Énée ("d'Airain ») et fait commencer sa fiction par ces deux mots énoncés de manière indissociable: Arma virumque... D'où l'épistémologie fonctionnelle qui caractérise la connaissance du Latin: c'est en modifiant le monde (dont il fait partie) que l'homme connaît et se connaît, et non simplement en l'observant et en construisant des hypothèses comme le fait le Grec. D'où aussi l'implication institutionnelle de ce mode d'investigation et la nécessité de recourir au droit pour en définir les limites éthiques. Cette tradition latine, très présente dans le droit, resurgit plus discrètement dans le champ de la scientificité moderne, laquelle s'énonce en termes philosophiques et, donc, selon des catégories grecques. Mais elle n'est pas insignifiante. Elle prend corps plutôt dans le monde anglosaxon (marqué par la sécularisation protestante) et dans des disciplines de transformation (la chimie pouvant être leur symbole), en attendant de se formuler dans des théories comme celles de l'évolutionnisme, du «big bang » ou du fractalisme. Mais la prédominance $\mathrm{du}$ modèle grec nous fait toujours envisager les disciplines technologiques et de transformation comme des "sciences appliquées" (même la physique théorique a longtemps été perçue comme une "mathématique appliquée »). Cette prégnance de l'épistémologie grecque, pour indépassable qu'elle soit, nous rend incapables de penser l'épistémologie latine à l'œuvre dans notre culture. Notre droit et notre éthique courent toujours derrière, sans jamais rattraper ni accompagner ni, encore moins, précéder les transformations du monde auxquelles nous procédons.

9 Ajoutons que, surdéterminant une épistémologie grecque qui, à l'époque hellénistique, tend à faire de la personne individuelle la véritable cité à construire, le discours chrétien latin a fait de la personne le matériau que, par une conversion permanente depuis la naissance (baptême) jusqu'à la mort, il s'agit de transformer d'homme pécheur en homme sauvé. 
10 Peut-être est-il opportun de faire remarquer que cette prise de position épistémologique qui définit aujourd'hui la discipline universitaire s'est établie avec l'Université, mais aussi contre elle. La logique de scientificité est bien l'une des caractéristiques essentielles de la démarche qui conduit à l'émergence des Universités aux $\mathrm{XII}^{\mathrm{e}}$ et $\mathrm{XIII}^{\mathrm{e}}$ siècles. Mais elle ne va pas jusqu'au bout. L'avènement d'un doctor, nouveau sujet d'énonciation du vrai grâce à une démarche de scientificité, ne rend pas caduc l'énoncé du vrai que détient l'institution croyante. La vérité scientifique, pour légitime qu'elle soit dans son ordre, est toujours considérée comme vassale de la vérité révélée. C'est en très grande partie hors de l'Université, gardienne de cette subordination, que s'élaborera l'émancipation et de la pensée et du sujet. Émancipation inachevée, car pour n'avoir jamais vraiment pris la foi révélée pour objet - laquelle n'est pas à confondre avec la croyance -, bien des disciplines universitaires, y compris dans le champ des sciences exactes, demeurent dans la figure d'une diction révélée de la vérité.

2. Discipline de formation

11 Synonyme de science, le terme de discipline n'est vraiment devenu vecteur principal du discours universitaire que lorsqu'il a assumé l'autre signification majeure qui va de pair avec la première: celle de formation. Une discipline universitaire est, de manière indissociable, une discipline de recherche et de formation. Cette conjonction est relativement récente. Elle ne date que d'une bonne centaine d'années. Elle aurait pu se produire plus tôt. L'élaboration de projets d'éducation pendant la Révolution française aurait déjà pu en fournir une excellente occasion, notamment lorsqu'il s'est agi de définir l'objet et les limites d'une éducation publique et que l'on a alors nettement distingué entre éducation nationale et instruction publique. Mais ce n'est qu'à la fin du $\mathrm{XIX}^{\mathrm{e}}$ siècle que le terme discipline est venu assumer l'enseignement en tant qu'instruction publique : d'abord au singulier, comme synonyme de "gymnastique de l'esprit [...], de développement du jugement, de la raison, de la faculté de combinaison et d'invention » (C. Hippeau, 1872), puis au pluriel, au sens de " matière d'enseignement susceptible de servir d'exercice intellectuel», comme l'écrit A. Chervel (Histoire de l'éducation, $\mathrm{n}^{\circ} 38,1988$ ) à qui nous devons le mérite d'avoir bien mis en évidence cette dénomination récente des enseignements scolaires et universitaires. A. Chervel estimait que le passage du singulier au pluriel ne s'était fait qu'au début du $x^{e}$ siècle, pour désigner d'un terme générique des enseignements alors répartis entre enseignements littéraires et enseignements scientifiques. Mais cette argumentation tombe. Nous avons en effet trouvé des attestations antérieures, dont la plus ancienne date de 1892, sous la plume de V. Basch. Dans celle-ci, les «disciplines scolaires ", qui caractérisent l'enseignement secondaire, ont pour objectif de «donner une forte instruction générale », qualifiée à la fois de "littéraire, philosophique et scientifique ». Moyen de "culture générale", de "culture intellectuelle et morale", cet « enseignement classique » " doit être dégagé de toute visée professionnelle », " ne doit rien viser, mais rendre apte à tout ».

Comme cette nouvelle acception du terme de disciplines n'a, semble-t-il, sur le moment donné lieu à aucun débat, nous en sommes réduits aux conjectures. Mais ce silence n'en est que plus révélateur. Quand un mot advient ou qu'un changement de sens se produit, qu'il est immédiatement accepté par tous, sans que l'on y prenne garde, au point d'évincer ou de rendre caducs les autres termes, l'événement sémantique n'en est que plus important. Nous sommes pour notre part enclin à voir dans cette qualification des 
enseignements secondaires et universitaires (ceux-ci n'étant pas institutionnellement coupés des premiers) un changement radical dans l'orientation générale donnée à l'éducation nationale. Pour la première fois, un régime républicain gouverne la France de manière durable. Or, à la différence d'un régime royaliste ou impérial, qui, avec l'aide de la religion, prétend détenir le bien, le vrai et le beau et entend, pour cette raison, inculquer ses vues à l'ensemble de la nouvelle génération, une République ne peut, par définition, imposer quoi que ce soit en matière de jugement quant à la morale, la vérité et l'esthétique. Ce jugement appartient uniquement à chacun de ceux qui la composent et en quoi elle consiste : les citoyens eux-mêmes. Mais pour que ceux-ci puissent porter un jugement qui ne soit pas l'effet de préjugés, de simples opinions ou d'intérêts, il est nécessaire que chacun soit "instruit». Le terme de discipline qui, jusqu'alors dans l'enseignement, désignait la police des établissements et la partie de l'éducation des élèves qui lui est liée, peut métaphoriquement convenir pour désigner cette " police » de l'esprit qu'est son instruction, à savoir sa soumission aux règles de la Raison, de la Méthode et de l'Analyse, dont la maîtrise est la garantie d'un jugement sain et libre. Une discipline est donc avant tout une logique procédurale, laquelle ne peut être qu'une logique de scientificité, à laquelle chaque individu, en tant que personne et sujet humain, soumet son intelligence de manière à instruire son jugement. Telle est la seule épistémologie acceptable pour une éducation en régime républicain, la seule aussi qui fonde l'autorité du maitre ou du professeur. Vecteurs de la forme scolaire et universitaire de l'éducation républicaine, les disciplines n'inculquent pas. Elles instruisent.

Certes, en raison de son usage chrétien et social, le terme reste chargé d'une lourde ambiguïté : la République n'est pas qu'une communauté de citoyens libres. C'est aussi concrètement une société composite, une nation, un État, une patrie. La religion, surtout dans sa tradition catholique, l'imprègne encore de ses valeurs. Les disciplines sont donc concrètement grosses de toutes ces références et implications. Faire de bons français patriotes, acculturer, sélectionner les meilleurs, préparer à certaines professions ou fonctions... nombreux et variés sont les objectifs réellement poursuivis par les enseignements, y compris universitaires, que vient qualifier le nouveau terme de disciplines. Théoriquement, il appartient à la logique disciplinaire de les mettre au jour et de porter sur eux un jugement critique. Objectif sans doute utopique, mais qui ne saurait pour autant être abandonné, sauf à déclarer que la société, telle qu'elle est, est auto-instituante, thèse qui, justement, sous-tend la logique curriculaire.

3. Le repli disciplinaire

14 Si cette logique curriculaire arrive sur l'Université comme une déferlante, c'est peutêtre aussi parce que l'Université n'assume plus véritablement sa mission. Le vecteur principal qui la porte, la logique de scientificité, finit en effet par se retourner contre lui-même. Paradoxe? Non. Une loi de la transmission. La logique de scientificité s'est autonomisée et, donc, institutionnalisée. Elle s'est progressivement coupée du sujet qui porte l'interrogation qu'elle instruit en la construisant. Plus exactement, elle a réduit le sujet à sa dimension épistémique, à sa capacité cognitive. Par définition, la démarche cognitive implique une spécification et un fractionnement indéfini. Toute interrogation sur le réel conduit à une investigation et à une réponse qui ne peuvent être que multiples et dissociées. Le jugement, c'est-à-dire l'appréciation et la décision, même temporaires et conditionnelles, que doit prendre le sujet ne sont pas contenues dans ces réponses. Elles n'en sont pas la somme. Elles les transcendent. Plus, donc, se ramifient les disciplines universitaires, légitimement portées par la logique de 
scientificité, plus se creuse l'écart entre les savoirs qu'elles construisent et le jugement qu'elles sont supposées instruire. L'écart se transforme en rupture, lorsqu'elles déclarent, sans aucune nuance, que le savoir, la production du savoir est leur seule finalité. Elles ne peuvent alors plus prétendre être des disciplines de formation, sauf s'il s'agit de formation à la recherche, et, encore, dans un sens réducteur, si la recherche ne repose plus sur une interrogation portée par le sujet-chercheur, mais par le projet de recherche lui-même.

Ce n'est pas tout. Comme le sujet étudiant vient à l'Université pour instruire son regard sur le monde et sur lui-même, instruction qui lui est très précieuse pour se construire comme acteur de sa propre autonomie, certes, mais aussi comme acteur du monde, c'est-à-dire comme professionnel, les disciplines universitaires laissent tenir, quand elles ne le font pas elles-mêmes, un discours sur ce rapport au monde pour le moins douteux, pour ne pas dire contradictoire. La relation entre les disciplines de recherche et le monde est énoncée en termes de théories et de pratiques, le lien entre les deux étant conçu comme une application. Le monde, et pas seulement celui de l'action, serait une application de la science! On se demande comment une telle bévue, avatar d'une conception prêtée par la tradition scolaire à Platon, a pu s'imposer au point de devenir une évidence indiscutable. Cela ne suffit point. On affirme même que l'application participe de la logique de la science, qu'elle est elle-même scientifique! C'est exactement le renversement et la négation de la démarche scientifique. C'est un modèle théologique sécularisé. La science, le scientifique se donnent dans la figure du Dieu de la Révélation, du Dieu qui crée le monde, qui crée par sa pensée et qui révèle sa création par sa parole. Est-il nécessaire de rappeler que la théorie est interne à la démarche heuristique et que, en dehors de ce processus, elle n'est rien? Est-il nécessaire de rappeler que le monde n'est pas une application de la science? Est-il nécessaire de rappeler qu'il n'y pas d'application savante de la science? Penser le contraire, c'est priver tout un chacun de la possibilité d'émettre un jugement légitime et le réserver au seul savant. Or, si le savant est qualifié pour instruire, selon une entrée spécifique, telle ou telle question, cette instruction est insuffisante pour produire le jugement qui la transcende. Même si, par hypothèse, tous les savants de toutes les disciplines étaient convoqués pour instruire une question donnée, le jugement à porter transcenderait encore la somme de leurs connaissances. Autre est le plan de la cognition, domaine de la scientificité, autre est le plan du jugement, de la décision et de l'action, domaine de la valeur, du vraisemblable et de l'intérêt. Il n'y a pas de « fondement théorique » à l'action!

La question de la didactique comme discipline illustre de manière on ne peut plus patente de ce repli et de ce retournement des disciplines universitaires sur ellesmêmes. Seules sont acceptables en France les didactiques des disciplines. Chaque discipline universitaire a sa didactique. Plus exactement, le groupe de disciplines universitaires qui a pour objet une matière qui donne aussi lieu à une discipline de l'enseignement secondaire (et désormais, hélas, primaire) développe sa propre didactique. La thèse qui justifie cette mainmise et donc l'interdiction de penser la question de l'enseignement/apprentissage comme une question universitaire et disciplinaire sui generis, est que l'enseignement d'une discipline scolaire est une application de la discipline universitaire correspondante. Comme celle-ci est plurielle, c'est donc à l'une d'entre elles que sera le plus souvent reconnue cette autorité matricielle et tutélaire. En langues, ce sera la linguistique (au singulier!). Cette conception, que nous refusons de qualifier de «théorie» tant elle en représente le 
contraire, procède d'une double substitution : la première est de substituer le savoir, dont la production est l'une des finalités des disciplines universitaires, à la formation du jugement qui est la finalité de la discipline scolaire. La seconde est de substituer, grâce à l'homologie, le savoir savant au savoir scolaire, la discipline savante à la discipline scolaire. Mais qui ne voit que cette double substitution aboutit à un retournement de la démarche scientifique, qu'elle conduit à concevoir l'enseignement scolaire (et universitaire ?) selon une logique d'inculcation, de révélation sécularisée ? Le savant (la Science) est dans la posture du Dieu qui détient le vrai (comme énoncé et non comme énonciation), le maître dans celle du transmetteur institutionnel (le clerc), l'élève dans celle du fidèle. Schéma que transforme en vulgate indiscutable, en le masquant à peine, le fameux triangle didactique (qui définit l'enseignement comme une mise en relation entre les trois pôles que sont le savoir, le maitre et l'élève) et que tente de justifier la non moins fameuse "théorie » de la transposition didactique, qui analyse la didactique comme la transformation des savoirs savants en savoirs enseignables et enseignés et comme la substitution des représentations savantes, exactes, indiscutables et « pures » aux représentations vulgaires, fausses et « impures ». Tel est le traitement que l'Université française a ré-servé à la question de la didactique, traitement on ne peut plus contraire à la logique sur laquelle elle entend se fonder. Les conséquences n'en sont pas que théoriques. Il a entraîné la création des IUFM, c'est-àdire l'autonomisation, par rapport aux Universités, de la formation aux disciplines académiques, à ces disciplines que, justement, l'Université considère comme son bien propre et sur lesquelles elle prétend maintenir sa tutelle. Autrement dit, il a laissé la formation aux disciplines scolaires à la logique qui lui est fondamentalement opposée : la logique curriculaire.

II. Curriculum

17 Le terme est ambigu à souhait. Ou, plutôt, il est d'une limpidité troublante. L'usage ordinaire du syntagme curriculum vitae pourrait laisser entendre que ce terme renvoie d'abord au parcours individuel de tout un chacun, parcours qui permet de se construire comme personne et comme individu dans la société. Mais tel n'est pas le sens premier que lui attribuent les savants qui l'ont utilisé pour décrire la formation d'un individu: s'ils ne rejettent pas le sens individuel, de soi assez peu formalisable, que nous lui reconnaissons dans la tradition française, ce n'est qu'en tant qu'il peut s'inscrire dans la «carrière » que la société propose aux individus, dans les trajectoires d'éducation formalisées que la société elle-même institue pour faire acquérir les compétences dont elle estime avoir besoin pour son propre développement. La logique qui le sous-tend fait du curriculum le vecteur de la forme scolaire de l'éducation sociétale. On ne sera donc pas surpris d'apprendre que ce nouveau « concept » de la formation vient d'Outreatlantique et qu'il s'oppose aux disciplines sur les points essentiels.

1. Un contre-modèle de la discipline

$18 \mathrm{Si}$ on les considère d'après leur logique interne, et non d'après ce qui se fait concrètement, car, on l'a vu, des retournements sont toujours possibles, le curriculum s'oppose à la discipline avant tout du point de vue épistémologique. La discipline a pour fonction première d'instruire un questionnement de la personne quant au vrai, de manière qu'elle puisse juger et décider "en connaissance de cause». L'autonomie disciplinaire repose sur l'autonomie de la personne, d'une manière indissociable, car c'est au niveau de la pensée, de l'autonomie du jugement que réside fondamentalement la liberté du sujet, de chaque sujet. Le sujet est donc premier. Mais ce sujet, est-il besoin de le souligner, ne se ramène ni au sujet épistémique, ni au sujet psychologique, ni au 
sujet de l'inconscient, ni au sujet acteur dans la société, ni à n'importe quel autre sujet que nos découpages savants ou autres peuvent distinguer. Il les est tous à la fois. Et, en tant que personne, il les transcende tous. Aussi est-il nécessaire qu'il soit instruit pour n'être pas le simple jouet de l'un d'entre eux en même temps que le jouet de son milieu. C'est donc à partir de ce sujet, à la fois singulier et collectif, autonome et solidaire, libre et responsable, que tout ce qu'impliquent les disciplines est envisagé. C'est d'abord de cela que témoigne le statut privilégié qui est reconnu à l'universitaire. Si les enseignants-chercheurs sont élus par leurs pairs et non directement nommés par une autorité institutionnelle, c'est parce que l'institution est totalement incompétente en matière de vérité (l'orthodoxie étant la négation même de la démarche de scientificité). Si les enseignants-chercheurs ont la responsabilité de charges administratives, ce n'est pas parce qu'ils sont plus compétents en ce domaine que les autres, c'est pour que l'institution universitaire soit bien finalisée par les missions de recherche et de formation qui sont les siennes, qu'elle soit bien portée par la logique disciplinaire et non pas mise au service d'une logique administrative qui, elle, serait nécessairement dépendante d'autres logiques : politiques, financières, idéologiques, sociales, etc. Si les enseignants-chercheurs ont la liberté de mener leurs recherches comme ils l'entendent, s'ils s'abstiennent de définir a priori les sujets de recherche de leurs étudiants de maîtrise ou de doctorat, ce n'est pas pour reconnaître un droit quelconque au caprice, mais bien parce que la logique de scientificité, malgré toutes les contraintes de l'appareillage méthodologique, ne peut arrêter a priori ni l'itinéraire ni, encore moins, la destination. Si tel était le cas, si le chemin et le résultat étaient connus d'avance, il n'y aurait plus de véritable recherche!

La logique curriculaire n'est pas républicaine, au sens où par République on n'entend moins un régime politique spécifique que l'assemblée des seuls individus qui la composent, la seule instance détentrice de la souveraineté et instituante des formes constitutives de l'organisation en société, que ces formes soient politiques (patrie, État, nation...) ou économiques (sociétés financières ou de production), religieuses, associatives, etc., autrement dit, l'instance qui soumet toutes ces instances internes aux principes que la seule raison - et non l'intérêt - impose et qui sont de soi universels. Le curriculum se définit à partir des attentes et des besoins de la société, telle qu'elle est dans sa diversité et ses changements. Il se complaît dans la démocratie, qui, si elle n'est pas républicaine, n'est qu'une démocratie d'intérêts et d'opinion, autrement dit une oligarchie ploutocratique et communicationnelle. L'individu n'y est pas considéré en tant que sujet personnel, mais, d'une part, en tant que sujet de désirs, de pulsions et de fantasmes, pour la satisfaction desquels il peut être prêt, sauf s'il est véritablement cultivé, à tout investir et dépenser, et, d'autre part, en tant que porteur de compétences possibles, si précieuses pour répondre, via la société de marché, aux appétences du premier. Dans ce jeu de l'un à l'autre, les individus sont forcément très inégaux. Ce n'est pas regrettable. C'est un fait, et un fait nécessaire, car c'est, pense-t-on, sur cette tension que repose le dynamisme créatif de la société, comme la force du courant électrique repose sur la différence de tension entre le pôle négatif et le pôle positif.

Dans cette conception démocratique de la société, la question de la vérité ne se pose plus. C'est une question oiseuse. Ce que nous tenons pour vrai n'est qu'une forme passagère et locale de représentation du monde. La vérité est, par nature, plurielle, diverse et changeante. Aussi est-il nécessaire que les individus, que la question du vrai inquiète et paralyse, appartiennent à des communautés locales, qu'ils soient non pas cultivés, mais acculturés, acculturation qui peut être plurielle (religieuse, politique, 
linguistique, professionnelle, ethnique, sexuelle, etc.). Cette pluralité d'appartenances et la liberté d'en changer sont même données comme la marque d'une authentique liberté et non celle d'un opportunisme inconsidéré et purement intéressé. L'engagement de demain ne devra rien à celui d'aujourd'hui, comme celui d'aujourd'hui ne doit rien à celui d'hier. Il n'y a même plus de sujet permanent, mais une succession d'états subjectifs. La question de la vérité n'est finalement qu'une question d'intérêt, tel que chacun le perçoit pour lui-même hic et nunc. Se donner la connaissance comme objectif est donc une utopie et, plus grave encore, une perte de temps inutile et nuisible. Seules peuvent être engagées les recherches dont la voie est déjà tracée et les résultats prévisibles à brève échéance. Car seule compte l'exploitation qui pourra en être faite, quel qu'en soit le domaine. La recherche et la formation qui lui est associée ne sont acceptables que dans cette perspective instrumentalisée. On se gardera cependant de ternir l'image de la recherche : dans notre culture actuelle, la scientificité est l'argument de "vente » et d'opinion de loin le plus puissant. On a besoin de son image pour faire accepter comme scientifiques des décisions et des projets qui, par définition, ne le sont pas et ne peuvent pas l'être. Le savant est donc requis pour cautionner, non pour contester. Même si, dans le même temps, on proteste avec toute l'énergie possible que l'on agit et que l'on décide avec un pragmatisme le plus absolu, c'est-à-dire loin de toute idéologie ou de toute théorie particulière, mais en s'attachant à la seule " vérité des faits".

2. Du projet d'établissement au référentiel

21 C'est cette conception des choses, avatar d'une conception libérale phagocytée par le capitalisme et se donnant même avec l'autorité de la science, la théorie socioconstructiviste, qui préside à une approche curriculaire de la formation et qui, après avoir absorbé les formations professionnelles, est en train d'engloutir des formations scolaires et s'en prend désormais aux formations universitaires. Les modalités en sont diverses. L'une est le projet d'établissement, qui s'appelle plan quadriennal dans l'Université. Le projet d'établissement, qui participe d'une logique de décentralisation, c'est-à-dire d'un transfert aux collectivités locales de la puissance étatique, change la nature de la relation entre les universitaires et l'autorité de tutelle. Dans une République, une administration jacobine peut tout à fait préserver l'autonomie disciplinaire. Décentralisée, la tradition jacobine se transforme nécessairement en jacobinisme personnel, puisque, le contrat se substituant à la loi, ce sont les responsables, les chefs, qui font la loi. Leurs volontés et leurs décisions s'imposent, que ce soit celle du Ministre de l'éducation nationale, du Préfet de région ou du Président d'Université. Ils ne sont plus les représentants de l'État. Chacun peut dire, comme l'a écrit Claude Allègre : «Ce que je veux» (Le Monde du 6 février 1998) et faire sien un pouvoir qu'il n'a pourtant reçu que par délégation républicaine. Dans ces conditions, le Président d'Université est moins un pair parmi les enseignants-chercheurs qu'un interlocuteur privilégié de l'autorité de tutelle (le Ministère) et des institutions territoriales, autorités conjointes. Chef d'établissement devant concevoir son rôle comme un chef d'entreprise, il est de moins en moins le représentant de l'ensemble des enseignants-chercheurs pour être le porteur de projets susceptibles d'être reconnus et financés par les payeurs, devenus décideurs.

Un projet de recherche n'est donc plus évalué selon sa pertinence épistémologique (qui n'appartient qu'aux chercheurs), mais selon sa pertinence " politique ». Il ne peut plus, par définition, être personnel. Il ne peut qu'émaner d'équipes. Plus fondamentalement encore, surtout dans les sciences humaines où le budget de fonctionnement et de 
recherche est ridiculement faible et où la marque institutionnelle (laboratoires et équipement) des disciplines est extrêmement ténue, les projets de recherche et de développement seront le fait d'une équipe de direction qui, composée de personnes de confiance, aura pour tâche de proposer, de convaincre et de manager. Les projets de recherche et de formation, par voie de conséquence, ne dépendront pas d'abord de ce que les enseignants-chercheurs peuvent souhaiter en fonction de leurs recherches ni même des recherches qui leur semblent être appelées par la situation présente de leurs disciplines, mais de l'adéquation qu'ils peuvent présenter avec les attentes et les besoins en termes de métiers ou de techniques que définissent les décideurs ou payeurs extérieurs. Une équipe n'existe et n'est financée que si elle est reconnue par le Ministère de tutelle. Ainsi donc, du moins en sciences humaines où il n'y a pas, par définition, de laboratoires dûment répertoriés, les enseignants-chercheurs relevant d'une même discipline, mais travaillant dans des domaines nettement distincts, puisqu'ils doivent couvrir le champ de la discipline enseignée, sont cependant obligés de se fédérer pour proposer des programmes de recherche dont la pertinence épistémologique est alors pratiquement nulle. Sauf cas particulier et momentané, les recherches de philosophes, d'historiens ou de littéraires portant sur l'antiquité ne recoupent pas celles de philosophes, d'historiens ou de littéraires qui portent sur le Moyen Âge, l'époque moderne ou l'époque contemporaine. En revanche, ils trouvent dans les autres Universités des collègues avec lesquels ils peuvent utilement confronter leurs travaux, mais avec lesquels ils ne peuvent faire équipe, puisqu'ils appartiennent à des établissements différents. Et s'ils ne s'astreignent pas à former une équipe de recherche, ils se mettent rapidement dans l'impossibilité d'en faire, alors même qu'ils ont été recrutés comme chercheurs : c'est à l'équipe et à son projet de recherche qu'est accordé le budget. L'approvisionnement des bibliothèques s'en trouve gravement affecté. Devenu irrégulier, il risque de les rendre à terme inutilisables, parce que beaucoup trop lacunaires. Il ne reste à l'enseignant-chercheur qu'à faire de la recherche sur ses ressources personnelles (acheter des livres et participer à des colloques). Ce qui est un comble. Comme des dispositifs complémentaires comme les écoles doctorales et la charte des doctorants contraignent le doctorant à appartenir à une équipe de recherche, même la possibilité de diriger de jeunes chercheurs lui sera bientôt interdite. Ceux-ci, du reste, ne pourront pas venir avec leur propre interrogation, leur propre projet, mais, comme dans les sciences exactes, devront réaliser le projet que l'« équipe" aura conçu pour eux, entraînant la mort de la recherche en sciences humaines, laquelle ne peut se renouveler que par le questionnement que porte le jeune chercheur. On ne pense pas par procuration. La méthodologie ne vaut pas épistémologie, l'appareillage la pensée, la technique la science. En sciences humaines, ce qui attire les meilleurs chercheurs qui viennent de l'étranger pour faire une thèse, c'est avant tout la notoriété d'un chercheur, la rencontre avec un penseur, non le dispositif.

De qui émane tout cet ensemble institutionnel et organisationnel qui se développe au sein de l'Université ? Au service de qui ou de quoi se met-il en place? La recherche fondamentale? La formation des étudiants selon les exigences épistémologiques des disciplines? Il suffit, pour y répondre, de considérer l'outil universel dont on se sert dans la logique curriculaire: le référentiel. Comme chacun sait, le référentiel est d'abord apparu pour définir les formations professionnalisantes. Puis il est entré progressivement dans le système scolaire, en particulier comme mode de rédaction des programmes des disciplines scolaires. Il est à la base de la création des IUFM (cf. le 
Rapport Bancel de 1989). Et il arrive dans les Universités, que ce soit sous la forme des grilles que le Ministère conçoit, sous le couvert de rationalisation, moins pour évaluer les besoins et les résultats des formations, que pour contraindre celles-ci à rentrer dans ses visées, ou que ce soit, en usage interne, d'un référentiel proprement dit, comme l'équipe présidentielle d'une Université en a ébauché un en juillet 1999 pour lancer la nouvelle campagne de contractualisation. Théoriquement, un référentiel complet se compose de quatre référentiels distincts : le référentiel professionnel proprement dit, qui est un descriptif analytique le plus complet de toutes les tâches et compétences requises pour leur bonne réalisation. De ce référentiel descriptif on déduit (!) un référentiel de formation, dont les principes d'organisation sont issus, par reprise ou par inversion, des formations scolaires. L'ensemble s'achève par un référentiel d'évaluation et un référentiel de certification, dont l'inspiration doit également beaucoup au système éducatif.

Mais ces emprunts ne doivent pas donner le change : la logique du référentiel est à l'opposé de celle des disciplines. Non seulement celles-ci y sont complètement instrumentalisées, mais elles sont même privées de leur logique propre et de leur autonomie. Ce qui intéresse, ce n'est pas l'instruction d'un sujet et de sa capacité de porter un jugement (ce qui est recherché par la fameuse dissertation «à la française "), c'est, bien au contraire, l'acquisition de compétences dont la maitrise appartient non pas à la personne elle-même, mais à son employeur (ce qu'inculque le dossier de synthèse). Le savoir est chosifié, normé. Il n'est qu'une compétence parmi d'autres savoir-faire et savoir-être. Le sujet est réduit à une somme de compétences, dont même l'évaluation lui échappe. Pour lui, «je» est un autre, un autre qui a autorité sur lui. Certes, la logique disciplinaire laisse beaucoup trop de choses dans l'implicite, mais elle invite à le mettre au jour et à le soumettre à l'examen. Mais ce que la logique curriculaire laisse dans l'implicite et interdit de critiquer, c'est l'autorité qui fonde l'élaboration des référentiels. En fait, c'est la société, qui s'y donne sous la figure de l'évidence, du fait indiscutable, sans aucune idéologie (ce que prétend affirmer le pragmatisme !). Mais quelle société ? La société organisée politiquement ? La société dont les instances internes ont été confrontées à l'instance républicaine pour avoir une fonction instituante? Ou seulement la société de marché, laquelle inclut et met à son service toute l'économie informationnelle dont l'École et Université devraient participer?

Privées de la formation initiale et continue des enseignants par la création des IUFM, à savoir du gros bataillon des formations qu'elles avaient pour mission d'assumer, les Universités qui sont constituées par les disciplines académiques (les sciences humaines et quelques disciplines scientifiques) sont donc désormais priées de s'investir dans la préparation à des métiers pour lesquels elles ne sont pas précisément " compétentes ». Leur survie est à ce prix. Ou, plutôt, leur survie est au prix des disciplines elles-mêmes, dont la logique est exclue par les critères dits rationnels qui président soit au financement des formations soit à la gestion des postes. Il est clair que, si les postes sont comptés et attribués uniquement sur un ratio effectif étudiants/effectif enseignants, toute discipline ne comptant pas un nombre suffisant d'étudiants sera démantelée et condamnée à disparaître à plus ou moins brève échéance. Une demande à délivrer un "DEA de sciences de l'éducation » totalement dédié aux multimédia, à l'exclusion des autres champs ou disciplines qu'implique la question de l'éducation, alors que ce champ intéresse avant tout les disciplines de l'information et de la communication, atteste, s'il en était besoin, que, désormais, ce n'est pas le projet 
scientifique qui compte, mais, dans une économie informationnelle, son habillage scientifique.

Vision manichéenne et purement fantasmatique ? Cette dernière démarche attesterait, s'il en était besoin, que les logiques ou paradigmes que nous cherchons à mettre en évidence, nécessairement schématiques et tranchés, sont bien à l'œuvre, y compris avec leur tendance à se retourner contre eux-mêmes lorsqu'ils se laissent emporter par leur propre dynamique. Aussi bien, le discours qui est à tenir n'est sans doute pas dans l'exclusion réciproque de ces deux paradigmes. Il est dans leur confrontation. Il importe que le discours universitaire, revenant sur son propre paradigme, sorte de la position de repli faussement sécuritaire qui est le sien et, mettant en œuvre la logique de scientificité qu'il maitrise, analyse le système curriculaire tel qu'il advient de l'extérieur et s'impose présentement aux formations scolaires et universitaires, le confronte avec tout l'implicite curriculaire présent dans la logique disciplinaire, fasse enfin des propositions, quant aux formations et aux recherches, qui prennent en compte les exigences légitimes des deux logiques. Ainsi les universitaires, en instruisant leur jugement personnel et le jugement de tous les acteurs, assumeront-ils pleinement la part de responsabilité qui est la leur.

\section{RÉSUMÉS}

Deux sortes de discours se confrontent à propos de l'Université française et, plus précisément, dans les Universités de sciences humaines : l'un, tenu en interne par les universitaires et fondé sur une logique disciplinaire; l'autre, tenu en externe par la société, fondé sur une logique curriculaire (socio-constructiviste) et porté par les milieux libéraux-capitalistes. Les changements institutionnels opérés par le second dans l'Université sont désormais tels que l'affrontement est inévitable et exige une clarification. Premières réflexions et analyses. 